\title{
The analysis of fluorophore orientation by multiphoton fluorescence microscopy
}

\author{
Jamie M. Leeder and David L. Andrews \\ School of Chemistry, University of East Anglia, Norwich, NR4 7TJ, U.K.
}

\begin{abstract}
The accessibility of tunable, ultrafast laser sources has spurred the development and wide application of specialized microscopy techniques based on chromophore fluorescence following two- and three-photon absorption. The attendant advantages of such methods, which have led to a host of important applications including three-dimensional biological imaging, include some features that have as yet received relatively little attention. In the investigation of cellular or subcellular processes, it is possible to discern not only on the location, concentration, and lifetime of molecular species, but also the orientations of key fluorophores. Detailed information can be secured on the degree of orientational order in specific cellular domains, or the lifetimes associated with the rotational motions of individual fluorophores; both are accessible from polarization-resolved measurements. This paper reports the equations that are required for any such investigation, determined by robust quantum electrodynamical derivation. The general analysis, addressing a system of chromophores oriented in three dimensions, determines the fluorescence signal produced by the nonlinear polarizations that are induced by multiphoton absorption, allowing for any rotational relaxation. The results indicate that multiphoton imaging can be further developed as a diagnostic tool, either to selectively discriminate micro-domains in vivo, or to monitor dynamical changes in intracellular fluorophore orientation.
\end{abstract}

Keywords: Two-photon, three-photon, multiphoton, fluorescence, microscopy, chromophore, fluorophore, optical polarization

\section{INTRODUCTION}

Fundamental for biological function, organic tissues naturally contain a high proportion of light-absorbing components (chromophores), many of which can emit detectable, often visible, light in response to the absorption of an ultraviolet input. The well-established technique of fluorescence microscopy utilizes the prevalence of such chromophores to identify cells and cellular components in vivo, generally exploiting a wavelength difference between the input and output photons - this difference typically reflecting energy losses due to a degree of internal vibrational relaxation. The application of short-wavelength ultraviolet input does, however, introduce a number of significant drawbacks in the imaging of organic samples, where generally the use of a high-energy, broadly absorbed input suffers from limited biological sample penetration, poor image resolution due to a high degree of output light scattering, and an increased risk of cellular photodamage. Whilst the issue of light scattering can be minimized by the use of confocal imaging microscopes that adopt an optical "pinhole" to suppress out-of-focus fluorescence, the following work addresses a more specialized fluorescence technique based on multiphoton molecular excitation, whose wide application and development is largely attributable to recent significant improvements in both the quality and accessibility of tunable, ultrafast laser sources.

In multiphoton fluorescence imaging, electronic excitation and subsequent fluorescence emission is initiated by the simultaneous absorption of two or more input photons, necessitating the use of high intensities. Many biological applications utilize the output of Ti:sapphire lasers, tunable sources emitting pulses of near-infrared light with the requisite high intensity, yet relatively low average power consumption. Since the input wavelength typically lies outside the absorption bands of most biological chromophores, and since long wavelength radiation also experiences much reduced scattering, the simultaneous absorption of multiple infra-red photons can be confined to a comparatively small cross-sectional area and focused deep into organic tissue. In consequence, the technique affords a capability for high 
contrast fluorescence imaging to sub-cellular resolution, with limited photodamage, and without any need to suppress light scattering. ${ }^{1-3}$ One of the most appealing features of multiphoton induced imaging is the inherent adaptability afforded by the process, particularly in choosing a suitable measurement modality. Alternative and complementary modes of measurement are now frequently combined with multiphoton fluorescence in imaging microscopy, including second-harmonic generation, sum-frequency generation, coherent anti-Stokes Raman scattering, and Raman spectroscopy - each interaction characterized by a distinct molecular photophysical origin. ${ }^{4,5}$ In general terms, the capture of high quality images resolved in three dimensions aids the investigation of cellular or sub-cellular systems, making it possible to locate and discern the concentration and structure of specific molecular species by fluorescence intensity distributions. Multiphoton techniques in particular also permit the investigation of reaction dynamics by a variety of means, such as through monitoring variations in the comparative orientations, densities and arrangements of the key chromophores that play an important role in determining cell and protein function. ${ }^{5-7}$ Detailed information on the degree of orientational order in specific cellular domains is accessible from polarization-resolved multiphoton measurements, the study of which appears to offer features that have yet to be fully explored in the context of biological imaging.

The following investigation concerns the detailed polarization properties of the fluorescence signal generated in response to one-, two- and three-photon excitation of a molecular chromophore system. Specifically, results that are established by means of an isotropic rotational average determine the induced fluorescence response generated within a fully disordered molecular environment; i.e. a complete system, or micro-domains in a complete system, within which all chromophores are randomly oriented in three dimensions relative to any external frame of reference. If, in a more ordered system, the degree of local orientational order within the system exhibits fluctuations, (either in consequence of a biological function or in response to a controlled external stimulus), an expected deviation from the determined isotropic fluorescence signal will quantifiably register the degree of departure from a fully disordered system. It can be anticipated that the averaged results will prove invaluable in determining the random orientation limit of a dynamic spectrum, providing a means by which multiphoton imaging can be further developed as a diagnostic tool to monitor and potentially quantify variations in intracellular chromophore orientation. Whilst the technique of three-photon induced fluorescence microscopy is less commonly studied in biological contexts than its two-photon counterpart, the former is also considered in the following analysis, since the intrinsic advantages afforded by multiphoton techniques in biological imaging - particularly enhanced image contrast - are expected to become more prominent as the number of concerted photon interactions increases. 8,9

In Section 2, necessary details relating to the processes of one-, two- and three-photon induced fluorescence are first established, determining each of the output signals in terms of their associated electric polarization and molecular transition moment properties through standard quantum electrodynamical (QED) methods. A subsequent analysis of the results through rotational averaging methods is presented in Section 3, followed in Section 4 by a brief discussion of potential applications and goals for future theoretical development.

\section{METHOD}

To generally approach the issues surrounding chromophore orientation or rotational freedom, it is appropriate to begin with a representation of an entire optical process that subsumes both the absorption of laser input and the emission of fluorescent radiation. Both stages occur with an efficiency that is determined by the strength of coupling between ground and excited state electronic levels, as determined by components of the relevant transition dipoles or multiphoton tensors. A detailed theoretical representation is therefore built on the basis of salient parameters delivered by quantum theory - specifically the quantum amplitudes for initial excitation and the fluorescent decay. In the language of QED, these quantum amplitudes are commonly termed 'matrix elements' as they are in principle derivable for any specified initial and final state; the process efficiency is proportional to the modulus square of the matrix element. With the radiation properly regarded as comprising quanta, absorption signifies the annihilation of one or more photons in the course of photoexcitation; emissive decay is associated with single-photon creation. In terms of efficiency, these processes are mutually independent and in practice occur in a step-wise fashion; for our purposes, rotational relaxation following light absorption can be assumed insignificant. To present results amenable to experimental measurement, matrix elements of the form $M_{f i}(\xi)$ are defined for electronic transitions between initial, $i$, and final, $f$, states in a 
fluorophore $\xi$. The output signal, $I_{f l u}^{(n)}(\phi)$, is a function of the (experimentally determined) angle between the polarization vector of the incident and emitted light $\phi$, and can be described in terms of matrix elements for both $n^{\text {th }}$ order multiphoton absorption and single-photon emission, namely $M_{\alpha 0}^{(n)}(\xi)$ and $M_{0 \alpha}(\xi)$ respectively - in which the molecular labels 0 and $\alpha$ correspond to molecular ground and excited states, i.e.:

$$
I_{f l u}^{(n)}(\phi)=\sum_{\xi} K^{(n)}\left\langle\left|M_{\alpha 0}^{(n)}(\xi)\right|^{2}\left|M_{0 \alpha}(\xi)\right|^{2}\right\rangle
$$

The fluorescence signal in equation (1) is thus portrayed in terms of the efficiencies of both the absorption and emission processes; the constant of proportionality $K^{(n)}$ is itself dependent on experimental parameters including the $n^{\text {th }}$ power of the mean laser irradiance and the degree of $n^{\text {th }}$ order coherence. To assess the relationship between $I_{f l u}^{(n)}$ and $\phi$ for a fully disordered system in which molecular chromophores (more specifically, the molecular transition moments associated with multiphoton absorption and single photon emission) are randomly oriented relative to the input propagation, the angular brackets in equation (1) are implemented in terms of a rotational average, the detailed procedure for which follows below. To determine the rotationally averaged results for one- and two-photon induced fluorescence, it is first necessary to define the form of all associated matrix elements, achieved through the standard methods of QED. ${ }^{10,11}$ Whilst the full derivation is to be presented in detail in a future publication, for present brevity it is appropriate to highlight just the important features of each matrix element, namely the relevant electric polarization and molecular transition moment parameters.

\subsection{One-Photon Induced Fluorescence}

The process of one-photon induced fluorescence embodies a development in terms of two distinct matter-radiation interactions. The first describes the optical excitation of a molecular centre by single-photon absorption, inducing an electronic transition from the molecular ground to an accessible excited state configuration. The second interaction describes the process of molecular relaxation and photon emission, returning the molecule to its pre-excitation arrangement. In terms of equation (1), the matrix elements for one-photon absorption and one-photon emission are required, where for the former:

$$
M_{\alpha 0}^{(1)}(\xi) \sim e_{i}^{(l)}(\boldsymbol{p}) \mu_{i}^{\alpha 0}
$$

In equation (2), utilizing $\boldsymbol{p}$ and $l$ to respectively represent the wave-vector and polarization of the input beam, $e_{i}^{(l)}(\boldsymbol{p})$ is the unit electric polarization vector - henceforth to be represented more concisely as $e_{i}^{(l)}$. These input parameters are distinct from $\boldsymbol{p}^{\prime}$ and $l^{\prime}$, where the prime is introduced to label properties of the output fluorescence, for example in the following matrix element for one-photon emission:

$$
M_{0 \alpha}(\xi) \sim \bar{e}_{i}^{\left(l^{\prime}\right)} \mu_{i}^{0 \alpha}
$$

In both equations (2) and (3), the molecular transition is, to a good approximation, expressible in terms of components of single transition dipole moments $\boldsymbol{\mu}^{\alpha 0}$ and $\boldsymbol{\mu}^{0 \alpha}$ respectively, where use of the ideal dipole approximation requires that all higher-order multipolar contributions are comparatively negligible. Substitution of equations (2) and (3) into equation (1) determines the fluorescence output for one-photon induced fluorescence as:

$$
I_{f l u}^{(1)}(\phi)=K^{(1)}\left\langle\left(S_{i j} \bar{S}_{k l} T_{i j} \bar{T}_{k l}\right)\right\rangle
$$


where summation over repeated subscript indices applies. For convenience, a new notation is now introduced in which the product of Cartesian components of the unit electric polarization vectors, and of the molecular transition moments, are incorporated into second rank tensors. Specifically, for example, $S_{i j}$ and $\bar{S}_{i j}$ are equivalent to $e_{i}^{(l)} \bar{e}_{j}^{\left(l^{\prime}\right)}$ and $\bar{e}_{i}^{(l)} e_{j}^{\left(l^{\prime}\right)}$. Likewise, the molecular transition moment products described by $T_{i j}$ and $\bar{T}_{i j}$ correspond to $\mu_{i}^{\alpha 0} \mu_{j}^{0 \alpha}$ and $\bar{\mu}_{i}^{\alpha 0} \bar{\mu}_{j}^{0 \alpha}$. In both cases, and in all subsequent applications of this notation, the last index in the electric polarization and molecular transition tensors relate to the process of one-photon emission.

\subsection{Two-Photon Induced Fluorescence}

The process of two-photon induced fluorescence is currently the most common, experimentally adopted form of multiphoton induced fluorescence. The overall process is characterized by three matter-radiation interactions - the concerted absorption at a given molecular site of two photons, followed by one-photon emission. Whereas the matrix element for one photon emission takes the same form as equation (3), the molecular transition from ground and excited states progresses through two separate one-photon absorptions; thus, the matrix element describing the multiphoton excitation accounts for a transition through a virtual intermediate state, represented as a weighted sum over all possible molecular intermediate states $r$ :

$$
M_{\alpha 0}^{(2)}(\xi) \sim e_{i}^{(\lambda)} e_{j}^{(\lambda)} \sum_{r} \frac{1}{\left(E_{0 r}+\hbar c p\right)}\left(\mu_{i}^{\alpha r} \mu_{j}^{r 0}+\mu_{j}^{\alpha r} \mu_{i}^{r 0}\right) .
$$

In practice, the energy denominator in equation (5) featuring $E_{0 r}$ - the difference between the initial and intermediate state energies - usually determines that one specific intermediate state will dominate over all other contributions, for example when $E_{0 r}$ converges on the negative photon energy $-\hbar c p$. The two dipole product contributions in the numerator of equation (5) relate to each of the two possible time-orderings in which the input photons can interact with the molecule, where for $\mu_{i}^{\alpha r} \mu_{j}^{r 0}$ a photon of polarization $e_{j}^{(\lambda)}$ interacts with the molecular centre before the photon of polarization $e_{i}^{(\lambda)}$ and vice versa in the $\mu_{j}^{\alpha r} \mu_{i}^{r 0}$ contribution. The above matrix element for two-photon absorption can be presented as:

$$
M_{\alpha 0}^{(2)}(\xi) \sim e_{i}^{(\lambda)} e_{j}^{(\lambda)} \alpha_{i j}^{\alpha 0},
$$

in which the second rank molecular response tensor $\alpha_{i j}^{\alpha 0}$ is utilized, the exact form of which is:

$$
\alpha_{i j}^{\alpha 0}=\sum_{r} \frac{1}{\left(E_{0 r}+\hbar c p\right)}\left(\mu_{i}^{\alpha r} \mu_{j}^{r 0}+\mu_{j}^{\alpha r} \mu_{i}^{r 0}\right) .
$$

Substitution of equations (3) and (6) into (1) determines the output fluorescence signal resulting from two-photon induced fluorescence as:

$$
I_{f l u}^{(2)}(\phi)=K^{(2)}\left\langle\left(S_{i j k} \bar{S}_{l m n} T_{i j k} \bar{T}_{l m n}\right)\right\rangle
$$

accommodating the electric polarizations and molecular transition moments within third rank tensors such that $S_{i j k}$ and $\bar{S}_{i j k}$ correspond to $e_{i}^{(l)} e_{j}^{(l)} \bar{e}_{k}^{\left(l^{\prime}\right)}$ and $\bar{e}_{i}^{(l)} \bar{e}_{j}^{(l)} e_{k}^{\left(l^{\prime}\right)}$, whilst both $T_{i j k}$ and $\bar{T}_{i j k}$ contain the second rank molecular response tensor as $\alpha_{i j}^{\alpha 0} \mu_{k}^{0 \alpha}$ and $\bar{\alpha}_{i j}^{\alpha 0} \bar{\mu}_{k}^{0 \alpha}$ respectively. 


\subsection{Three-Photon Induced Fluorescence}

The output signal for three-photon induced fluorescence is dependent on the rotational average of the combined matrix elements of three-photon absorption and one-photon emission, the former expressible as:

$$
M_{\alpha 0}^{(3)}(\xi) \sim e_{i}^{(\lambda)} e_{j}^{(\lambda)} e_{k}^{(\lambda)} \beta_{i j k}^{\alpha 0},
$$

featuring the third rank molecular response tensor $\beta_{i j k}^{\alpha 0}$ as:

$$
\beta_{i j k}^{\alpha 0}=\sum_{r, s}\left(\mu_{i}^{\alpha s} \mu_{j}^{s r} \mu_{k}^{r 0}+\mu_{i}^{\alpha s} \mu_{k}^{s r} \mu_{j}^{r 0}+\mu_{j}^{\alpha s} \mu_{i}^{s r} \mu_{k}^{r 0}+\mu_{j}^{\alpha s} \mu_{k}^{s r} \mu_{i}^{r 0}+\mu_{k}^{\alpha s} \mu_{i}^{s r} \mu_{j}^{r 0}+\mu_{k}^{\alpha s} \mu_{j}^{s r} \mu_{i}^{r 0}\right)\left(E_{0 r}+\hbar c p\right)^{-1}\left(E_{0 s}+2 \hbar c p\right)^{-1}
$$

The inclusion of an additional photon-matter interaction introduces an additional intermediate state $s$, the matrix element now being duly summed over all possible $r$ and $s$ intermediates. The fluorescence output due to three-photon induced fluorescence is therefore presentable as:

$$
I_{f l u}^{(3)}(\phi)=K^{(3)}\left\langle\left(S_{i j k l} \bar{S}_{m n o p} T_{i j k l} \bar{T}_{\text {mnop }}\right)\right\rangle
$$

Here, following previous procedures, the electric polarization and molecular transition moments have been expressed in terms of fourth rank molecular tensors, the final index of each again being associated with the one-photon emission.

\section{RESULTS}

The general form of result for the fluorescence output in one-, two- and three-photon induced systems, as represented by equations (4), (8) and (11), respectively, is applicable to arbitrary orientations of the responsible fluorophores with respect to experimentally determined input and detection configurations. As such, these results are in principle directly applicable to samples with individual fluorophores in a fixed orientation, or those comprising local fluorophore domains with orientational correlation. To address less ordered systems it is expedient to also secure corresponding results for the other extreme - systems of completely random orientation. To this end, the above results are now subject to a rotation averaging protocol. The one-photon induced fluorescence system is addressed first, highlighting the important procedures within the method in detail - although the simplicity of this case belies significant technical complexity in securing results for the higher order interactions.

\subsection{One-Photon Induced Fluorescence}

From equation (4), the one-photon induced fluorescence signal is clearly dependent upon four separate Cartesian indices, each of which can assume $x, y$ or $z$ values with respect to a chosen frame. In consequence the electric polarization and molecular transition moment parameters within the angular brackets are resolved through fourth-rank rotational averaging. The first step is to decouple both the molecular and radiation components of the system, arbitrarily assigning a laboratory-fixed frame of reference, denoted by Latin indices, to the electric polarization parameters - such that the components of both $S_{i j}$ and $\bar{S}_{k l}$ become independent of molecular orientation. The molecular transition moments within the molecular tensors $T_{i j}$ and $\bar{T}_{k l}$ are subsequently associated to a molecule-fixed reference frame, labeled by Greek indices, i.e.:

$$
I_{f l u}^{(1)}(\phi)=K^{(1)}\left\langle\left(S_{i j} \bar{S}_{k l} T_{i j} \bar{T}_{k l}\right)\right\rangle=K^{(1)}\left(S_{i j} \bar{S}_{k l} T_{\lambda \mu} \bar{T}_{v \sigma}\left\langle\ell_{i \lambda} \ell_{j \mu} \ell_{k v} \ell_{l \sigma}\right\rangle\right)
$$


where it is now only the direction cosines, linking the two reference frames (for example $\ell_{i \lambda}$ which represents the cosine of the angle between the molecular orientation axis, $\lambda$ relative to the fixed radiation axis, $i$ ) that are dependent on molecular orientation. The generalized result of the fourth-rank average follows: ${ }^{10,12}$

$$
\left\langle\ell_{i \lambda} \ell_{j \mu} \ell_{k v} \ell_{l o}\right\rangle=\frac{1}{30}\left(\begin{array}{l}
\delta_{i j} \delta_{k l} \\
\delta_{i k} \delta_{j l} \\
\delta_{i l} \delta_{j k}
\end{array}\right)^{T}\left(\begin{array}{ccc}
4 & -1 & -1 \\
-1 & 4 & -1 \\
-1 & -1 & 4
\end{array}\right)\left(\begin{array}{l}
\delta_{\lambda \mu} \delta_{v \sigma} \\
\delta_{\lambda v} \delta_{\mu \sigma} \\
\delta_{\lambda \sigma} \delta_{\mu v}
\end{array}\right),
$$

in which the products of the Kronecker deltas act to transform the indices within the right hand side of equation (12). Following all such transformations on substitution of equation (13) into equation (12), it is necessary to consider the effects of applied summation over repeated indices. In the radiation frame, the resultant form of the electric polarization tensors is dependent on the polarization properties of the input laser light. In the commonly utilized deployment of plane-polarized input laser light the product of any two polarization vectors from the same beam yields unity, whilst all products of an input and output polarization vector are expressible as the cosine of the angle $\phi$ between them:

$$
\begin{aligned}
& \left(\mathbf{e}^{(\lambda)} \cdot \overline{\mathbf{e}}^{(\lambda)}\right),\left(\mathbf{e}^{\left(\lambda^{\prime}\right)} \cdot \overline{\mathbf{e}}^{\left(\lambda^{\prime}\right)}\right)=1 \\
& \left(\mathbf{e}^{(\lambda)} \cdot \mathbf{e}^{(\lambda)}\right),\left(\overline{\mathbf{e}}^{(\lambda)} \cdot \overline{\mathbf{e}}^{(\lambda)}\right),\left(\mathbf{e}^{\left(\lambda^{\prime}\right)} \cdot \mathbf{e}^{\left(\lambda^{\prime}\right)}\right),\left(\overline{\mathbf{e}}^{\left(\lambda^{\prime}\right)} \cdot \overline{\mathbf{e}}^{\left(\lambda^{\prime}\right)}\right)=1 \\
& \left(\mathbf{e}^{(\lambda)} \cdot \mathbf{e}^{\left(\lambda^{\prime}\right)}\right),\left(\mathbf{e}^{(\lambda)} \cdot \overline{\mathbf{e}}^{\left(\lambda^{\prime}\right)}\right),\left(\overline{\mathbf{e}}^{(\lambda)} \cdot \mathbf{e}^{\left(\lambda^{\prime}\right)}\right),\left(\overline{\mathbf{e}}^{(\lambda)} \cdot \overline{\mathbf{e}}^{\left(\lambda^{\prime}\right)}\right)=\cos \phi,
\end{aligned}
$$

hence equation (12) is now expressible in terms of $\phi$ as:

$$
I_{f l u}^{(1)}(\phi)=\frac{K^{(1)}}{30}\left[\left(T_{\lambda \lambda} \bar{T}_{\mu \mu}+T_{\lambda \mu} \bar{T}_{\mu \lambda}\right)\left(3 \cos ^{2} \phi-1\right)-\left(2 T_{\lambda \mu} \bar{T}_{\lambda \mu}\right)\left(\cos ^{2} \phi-2\right)\right],
$$

signifying the rotationally averaged fluorescence output from one-photon induced fluorescence.

\subsection{Two-Photon Induced Fluorescence}

The previously established averaging methods are now applied to two-photon induced fluorescence by identical means, beginning with the expression for the output fluorescence signal, equation (8) and decoupling the molecular and radiation frames as before:

$$
I_{f l u}^{(2)}(\phi)=K^{(2)}\left(S_{i j k} \bar{S}_{l m n} T_{\lambda \mu \nu} \bar{T}_{\sigma \tau \rho}\left\langle\ell_{i \lambda} \ell_{j \mu} \ell_{k v} \ell_{l \sigma} \ell_{m \tau} \ell_{n \rho}\right\rangle\right)
$$

the implementation of which is resolvable through a sixth-rank average. The resolution of equation (16) invokes considerably greater calculational complexity, owing to the fact that the generalized expression $\left\langle\ell_{i \lambda} \ell_{j \mu} \ell_{k \nu} \ell_{l \sigma} \ell_{m \tau} \ell_{n \rho}\right\rangle$ is notably larger than the fourth-rank equivalent of equation (13) - the exact form of the former referenced elsewhere due to present space constraints. ${ }^{10,12}$ In fact, it transpires that the fluorescence signal is expressible generally in terms of 15 distinct molecular invariants (compared to 3 in the one-photon case), each necessarily summed over all intermediate states $r$ such that: 


$$
\begin{aligned}
I_{f l u}^{(2)}(\phi)= & \frac{K^{(2)}}{210}\left[\left(T_{\lambda \lambda \mu} \bar{T}_{\mu v v}+T_{\lambda \lambda \mu} \bar{T}_{v \mu v}+T_{\lambda \mu \lambda} \bar{T}_{\mu v v}+T_{\lambda \mu \lambda} \bar{T}_{v \mu v}+T_{\lambda \mu \nu} \bar{T}_{v v \mu}+T_{\lambda \mu \mu} \bar{T}_{\lambda v v}+T_{\lambda \mu v} \bar{T}_{\lambda v \mu}+T_{\lambda \mu \mu} \bar{T}_{v \lambda v}+T_{\lambda \mu v} \bar{T}_{\nu \lambda \mu}\right.\right. \\
& \left.\left.+T_{\lambda \mu \mu} \bar{T}_{v v \lambda}+T_{\lambda \mu v} \bar{T}_{\mu v \lambda}+T_{\lambda \mu v} \bar{T}_{v \mu \lambda}\right)\left(3 \cos ^{2} \phi-1\right)-2\left(T_{\lambda \lambda \mu} \bar{T}_{v v \mu}+T_{\lambda \mu v} \bar{T}_{\lambda \mu v}+T_{\lambda \mu v} \bar{T}_{\mu \lambda v}\right)\left(2 \cos ^{2} \phi-3\right)\right] .
\end{aligned}
$$

It is however possible to present equation (17) in a more concise form, without compromising the accuracy or generality of the result, by considering index symmetry properties. Since the product of unit electric polarization components in the two-photon absorption matrix element is symmetric with respect to a permutation of the Cartesian indices, only molecular transition moment products that are $i-, j$ - symmetric can deliver non-vanishing contributions to equation (7). Hence, index-symmetrized forms of the molecular tensors in equation (17) can be invoked, where some of the molecular invariant pairs become identical, leading for example to the substitution $T_{\lambda \lambda \mu} \bar{T}_{\mu \nu v}+T_{\lambda \lambda \mu} \bar{T}_{\nu \mu \nu} \equiv 2 T_{(\lambda \lambda) \mu} \bar{T}_{(\mu \nu) v}$. Overall, the 15 molecular invariants thereby reduce to just 6 distinct terms:

$$
I_{f u u}^{(2)}(\phi)=\frac{K^{(2)}}{105}\left[\left(T_{(\lambda \lambda) \mu} \bar{T}_{(\mu \nu) v}+2 T_{(\lambda \mu) \lambda} \bar{T}_{(\mu \nu) v}+T_{(\lambda \mu) \lambda} \bar{T}_{(\nu v) \mu}+2 T_{(\lambda \mu) v} \bar{T}_{(\lambda v) \mu}\right)\left(3 \cos ^{2} \phi-1\right)-\left(T_{(\lambda \lambda) \mu} \bar{T}_{(\nu v) \mu}+2 T_{(\lambda \mu) v} \bar{T}_{(\lambda \mu) v}\right)\left(2 \cos ^{2} \phi-3\right)\right] .
$$

Whilst expressions of similar form to equation (15) have been presented previously, for example by Andrews and Allcock as early as $1995,{ }^{13}$ the equivalent result for two-photon induced fluorescence as represented above has to our knowledge not been established before.

\subsection{Three-Photon Induced Fluorescence}

Finally, the result of three-photon induced fluorescence is considered, beginning from the re-expression of equation (11) as:

$$
I_{f l u}^{(3)}(\phi)=K^{(3)}\left(S_{i j k l} \bar{S}_{m \text { mop }} T_{\lambda \mu v \sigma} \bar{T}_{\tau \rho \pi \eta}\left\langle\ell_{i \lambda} \ell_{j \mu} \ell_{k v} \ell_{l \sigma} \ell_{m \tau} \ell_{n \rho} \ell_{o \pi} \ell_{p \eta}\right\rangle\right),
$$

requiring an eighth-rank average. Compared to the fourth and sixth rank rotational averages utilized earlier, the general form of the eighth-rank, specifically applied as $\left\langle\ell_{i \lambda} \ell_{j \mu} \ell_{k \nu} \ell_{{ }_{\sigma}} \ell_{m \tau} \ell_{n \rho} \ell_{o \pi} \ell_{p \eta}\right\rangle$ is rarely reported owing largely to the extreme complexity in presenting and resolving the matrix equation, first derived by Andrews and Ghoul. ${ }^{14}$ Similarly, due to present size constraints, the full matrix equation necessary to resolve three-photon induced fluorescence is not presented here. However, following the same methodology utilized in the previous two examples, a result has now been determined. The result for plane-polarized input is specifically shown to generate a fluorescence signal whose properties are described in terms of 105 distinct molecular invariants. In order to present a manageable result, it is necessary to again invoke full index symmetry within the molecular multiphoton excitation parameters, notably the third-rank molecular response tensor, $\beta_{i j k}^{\alpha 0}$ allowing the output signal to be expressed in terms of just eight unique molecular invariants:

$$
\begin{aligned}
I_{f l u}^{(3)}(\phi)= & \frac{K^{(3)}}{630}\left[\left(3 T_{(\lambda \lambda \mu) \mu} \bar{T}_{(\nu v \sigma) \sigma}+6 T_{(\lambda \lambda \mu) v} \bar{T}_{(\mu \nu \sigma) \sigma}+3 T_{(\lambda \mu \mu) v} \bar{T}_{(\nu \sigma \sigma) \mu}+6 T_{(\lambda \mu \nu) \lambda} \bar{T}_{(\mu \nu \sigma) \sigma}+6 T_{(\lambda \mu \nu) \lambda} \bar{T}_{(\mu \sigma \sigma) v}+6 T_{(\lambda \mu \nu) \sigma} \bar{T}_{(\lambda \mu \sigma) v}\right)\left(3 \cos ^{2} \phi-1\right)\right. \\
& \left.-\left(6 T_{(\lambda \lambda \mu) v} \bar{T}_{(\mu \sigma \sigma) v}+4 T_{(\lambda \mu v) \sigma} \bar{T}_{(\lambda \mu \nu) \sigma}\right)\left(3 \cos ^{2} \phi-4\right)\right] .
\end{aligned}
$$

Again, it is worth highlighting that this result appears not to have been achieved or reported previously. It is also worth noting that, without the matrix method of securing rotationally averaged results, the calculation would entail no less than $3^{16}=43,046,721$ separate trigonometric integrals (each Latin and each Greek index in equation (19) taking one of three independent Cartesian values). 


\section{DISCUSSION}

By determining how a multiphoton-induced fluorescence signal responds to a controlled variable - in particular the angle of a signal polarizer - it may prove possible to distinguish, and more importantly quantify, any variation in localized orientational disorder within a bulk sample. Such variations would be manifest as deviations from the expected response. From a theoretical viewpoint, our initial results show promise, the equations that determine the required multiphoton fluorescence response, namely equations (18) and (20), proving to be expressible in a relatively simple form. It is worth highlighting a significant trend in all of the results through comparison of equations (15), (18) and (20). It emerges that the fluorescence outputs associated with randomly disposed fluorophores can be described through the following generalized formula:

$$
I_{f l u}^{(n)}(\phi)=K^{(n)}\left(A^{(n)}\left(3 \cos ^{2} \phi-1\right)-B^{(n)}\left(n \cos ^{2} \phi-(n+1)\right)\right),
$$

with both $A^{(n)}$ and $B^{(n)}$ representable as a sum of distinct molecular invariants. Clearly, in each case of single- and multiphoton induced fluorescence, the measured signal is dependent on two separate functions of the angle $\phi$. Of particular interest to the experimentalist will be the occurrence of the second Legendre polynomial $\left(3 \cos ^{2} \phi-1\right)$, a function commonly encountered in the fields of solid-state nuclear magnetic resonance and time-resolved fluorescence anisotropy. ${ }^{15,16}$ Both areas of study commonly exploit "magic angle" measurements, made under conditions where $\phi$ is roughly $54.7^{\circ}$, in which case terms dependent on $3 \cos ^{2} \phi-1$ clearly disappear. Multiphoton induced fluorescence measurements made at the magic angle of polarization analysis would therefore enable the identification, at least in relative terms, of $B^{(n)}$; analysis under other conditions would then lead to a corresponding value for $A^{(n)}$. By varying the angle $\phi$ it should be relatively straightforward to determine the general degree of conformity to equation (21), departures from which quantifiably signify domains of local orientational correlation. It is noteworthy that there is no angle at which the $B^{(n)}$ term can be made to vanish. The key to completely identifying the character of local order is likely to involve a series of experimental measurements with not only the orientation of the polarization resolver being varied, but also the sample itself being rotated laterally. The search for a robust protocol based on such a methodology is the subject of currently ongoing research.

\section{REFERENCES}

[1] Rubart, M., “Two-Photon Microscopy of Cells and Tissue,” Circ. Res. 95, 1154-1166 (2004).

[2] Diaspro, A., Bianchini, P., Vicidomini, G., Faretta, M., Ramoino, P., and Usai, C., "Multi-photon excitation microscopy," Biomed. Eng. Online 5, 36-49 (2006).

[3] Oheim, M., Michael, D. J., Geisbauer, M., Madsen, D., and Chow, R. H., "Principles of two-photon excitation fluorescence microscopy and other nonlinear imaging approaches," Adv. Drug Deliver. Rev. 58, 788-808 (2006).

[4] Jhan, J., Chang, W., Chen, H., Lee, Y., Wu, M., Chen, C., and Liau, I., "Integrate multiple multi-photon imaging and Raman spectroscopy for characterizing structure-constituent correlation of tissues," Opt. Express 16, 1643116441 (2008).

[5] Provenzano, P. P., Eliceiri, K. W., and Keely, P. J., "Multiphoton microscopy and fluorescence lifetime imaging microscopy (FLIM) to monitor metastasis and the tumor microenvironment," Clin. Exp. Metastasis 26, 357-370 (2009).

[6] Gratton, E., Barry, N. P., Beretta, S., and Celli, A., “Multiphoton Fluorescence Microscopy,” Methods 25, 103-110 (2001).

[7] Lee, P. F., Yeh, A. T., and Bayless, K. J., "Nonlinear optical microscopy reveals invading endothelial cells anisotropically alter three-dimensional collagen matrices," Exp. Cell Res. 315, 396-410 (2009).

[8] Balaji, J., Desai, R., and Maiti, S., "Live cell ultraviolet microscopy: A comparison between two- and three-photon excitation,” Microsc. Res. Techniq. 63, 67-71 (2004).

[9] Chu, S. W., Tai, S. P., Ho, C. L., Lin, C. H., and Sun, C. K., "High-resolution simultaneous three-photon fluorescence and third-harmonic-generation microscopy," Microsc. Res. Techniq. 66, 193-197 (2005).

[10] Craig, D. P., and Thirunamachandran, T., [Molecular Quantum Electrodynamics], Dover, New York (1998). 
[11] Salam, A., "Molecular quantum electrodynamics in the Heisenberg picture: A field theoretic viewpoint," Int. Rev. Phys. Chem. 27, 405-448 (2008).

[12] Andrews, D. L., and Thirunamachandran, T., “On three-dimensional rotational averages,” J. Chem. Phys. 67, 50265033 (1977).

[13] Andrews, D. L., and Allcock, P., "Polarization analysis of bimolecular excitations mediated by energy transfer: A common theoretical framework for fluorescence migration and sequential Raman scattering," Chem. Phys. 198, 35 51 (1995).

[14] Andrews, D. L., and Ghoul, W. A., "Eighth rank isotropic tensors and rotational averages," J. Phys. A: Math. Gen. 14, 1281-1290 (1981).

[15] Geddes, C. D., and Lakowicz, J. R. eds., [Reviews in Fluorescence 2005], Springer, New York (2005).

[16] Alia, A., Ganapathy, S., and Groot, H. J., "Magic angle spinning (MAS) NMR: A new tool to study the spatial and electronic structure of photosynthetic complexes," Photosynth. Res. 102, 415-425 (2009). 